\title{
Salicylic acid: old and new implications for the treatment of type 2 diabetes?
}

\author{
Graham Rena $\cdot$ Kei Sakamoto
}

Received: 1 May 2014/ Accepted: 14 May 2014/Published online: 12 June 2014

(C) The Japan Diabetes Society 2014

\begin{abstract}
Efficacy of salicylic acid as a treatment for diabetes was first established well over a century ago. Antihyperglycaemic effects are thought to include improved peripheral insulin sensitivity and suppression of hepatic glucose production. For most of this period, the molecular mechanisms underlying these effects have been poorly understood and these are still a focus of considerable research, which is reviewed here. Antihyperglycaemic effects are observed only at much higher concentrations than analgesic, antipyretic and antithrombotic properties, suggesting that different targets underlie the antidiabetic aspects of salicylate pharmacology. In the 1950s, antihyperglycaemic responses were linked to mitochondrial uncoupling effects of the drug. Then at the beginning of this century, antihyperglycaemic effects were linked to anti-inflammatory effects of the drug on NF- $\kappa B$ signalling. More recently, new work suggests that direct activation of AMPK may contribute to antihyperglycaemic/antihyperlipidemic actions of salicylates. Better understanding of the mechanism of salicylate's anthyperglycaemic effects may ultimately accelerate the development of new drugs for human use.
\end{abstract}

Keywords Salicylate $\cdot$ Aspirin $\cdot$ Salsalate .

Inflammation $\cdot \mathrm{NF}-\kappa \mathrm{B} \cdot \mathrm{AMPK}$

G. Rena $(\square)$

Cardiovascular and Diabetes Medicine, Ninewells Hospital and Medical School, University of Dundee, Dundee DD1 9SY, UK

e-mail: g.rena@dundee.ac.uk

K. Sakamoto ( $\square)$

Nestlé Institute of Health Sciences SA, EPFL Innovation Park, 1015 Lausanne, Switzerland

e-mail: Kei.Sakamoto@rd.nestle.com

\section{Ancient and early studies}

Empirical therapeutic use of naturally occurring salicylatelike compounds, abundant in plants such as myrtle and willow [1], has been ongoing since the early days of civilization in most parts of the world. For example, use of the willow is included in many early medical texts, including a Sumerian tablet dated to the third millennium B.C.E. [2]. Even though the active agent was unknown until much later, some of the early applications suggested for salicylate-containing plants were appropriate by modern standards. The Edwin Smith Surgical Papyrus from ancient Egypt [3] recommends use of willow leaves as 'a cool medium for drawing the warmth,' when a wound 'is inflamed, with a concentration of warmth spewing from the mouth of that wound, and the lips of the wound are ruddy [red] and when that man is feverish from it.' The text illustrates not only that some of the cardinal signs of inflammation were recognized, but also suggests that antiinflammatory/anti-septic properties of willow were known [3]. Another text, The Hearst Medical Papyrus, recommends willow to 'cool' chronic inflammation likely to be arthritis or rheumatism [4]. By the time of ancient Greece and Rome, salicylate-containing plants were used for their analgesic, keratolytic, anti-inflammatory and antiseptic properties [5-7].

In the nineteenth century, there were significant advances in the development of the salicylates as drugs. It became possible to isolate salicylate-related active agents such as salicin from plant extracts [7] and study them clinically. Perhaps the first such (uncontrolled) study was performed by MacLagan, a Dundee physician, who established that salicin, which is converted to salicylate, is a useful treatment for rheumatic fever [8]. Alongside this work on the natural products, Kolbe and 
Lauteman devised a chemical synthesis of salicylate that is still used today [6,7], enabling salicylate to be synthesized in increasing quantities and allowing the therapeutic effects of this drug to be studied in isolation for the first time. At about the same time as MacLagan's work on salicin, for example, Stricker reported that salicylate was also an effective treatment for rheumatic fever [9].

Salicylate itself is used little today and one of the main reasons for this is the identification by Bayer of a superior compound, acetylsalicylic acid (aspirin) [7]. This agent largely replaced salicylate use in the clinic and went on to become one of the most widely used medications. Mechanisms of action of salicylate and aspirin only began to be established much later, once the intracellular targets of the drug were studied, culminating in Vane sharing the Nobel prize for the discovery that aspirin blocks the formation of arachidonic acid-derived inflammatory mediators [10].

In relation to diabetes, antihyperglycaemic effects of salicylate began to be reported in the 1870s. Gross and Greenberg covered this early period well in their comprehensive monograph published in 1948 [6]. Starting with the work of Ebstein [11] and including the first detailed English-language account by Williamson in 1901 [12], they cite 15 clinical reports of antihyperglycaemic effects of salicylate [6]; however, at the time they were writing, Gross and Greenberg reported that this use had long since been discontinued. Independently, the effect was in the process of being rediscovered experimentally, when it was found that aspirin, which is metabolized to salicylate $[6,13]$, was capable of inducing antihyperglycaemic effects in partly depancreatised animals [14]. Salicylate was also effective in alleviating hyperglycaemia following alloxan treatment [15]. In the same decade, Reid, MacDougall and Andrews correctly deduced and then investigated similar actions in humans, when confronted with unexpected normoglycemia in a known diabetes patient who was taking only aspirin as part of a rheumatic fever trial [13]. These early studies established not only that larger (gram) quantities of the drugs were required than were necessary for analgesic/antipyretic effects [6, 12-14], but also that salicylate did not induce hypoglycaemia [13]. Unfortunately, at the doses used, the beneficial effects of salicylate were accompanied by serious side effects, including persistent nausea, vomiting, tinnitus and deafness [13]. The nausea and vomiting in particular could in turn give rise to starvation ketosis, which was hard to distinguish from diabetic ketosis and which was thought to have accounted for the salicylates' abandonment in clinical use [16].

\section{Effects on mitochondrial function/ATP production}

The rediscovery in the 1950s of salicylate's effects on glucose metabolism prompted investigation of the mechanism. One study found increased oxygen consumption in humans given doses either of $10 \mathrm{~g}$ salicylate intravenously or 7.5-10 g aspirin orally [17]. Elevation of oxygen consumption was also observed in rats [18] and rat diaphragm [19]. Shortly afterwards, using tissues and isolated mitochondria, it was found that salicylate uncoupled oxygen consumption from generation of ATP [20-22]. The effects of salicylate on metabolism were qualitatively similar to the uncoupler 2,4 dinitrophenol (DNP), in that both drugs increased oxygen consumption and glycogen breakdown, while decreasing ATP production [19-26]. In addition, effects of salicylate on mitochondrial oxygen production correlated well with antihyperglycaemic effects, because neither property was shared by other hydroxybenzoate analogues of the drug, such as 2,5 diHBA (also known as gentisate) and 2,6 diHBA (also known as $\gamma$-resorcylate) $[15,27,28]$. In tissues, it was found that salicylate and DNP increased glucose uptake in isolated rat diaphragm and heart muscle [29-31], while later studies in perfused liver found that salicylate inhibited hepatic gluconeogenesis [32]. More recent work on aspirin used clamps and glucose tracers to study whole body glucose disposal. These studies confirmed that blood glucose is lowered through a combination of reduced hepatic glucose production and elevated glucose uptake [33].

The work just described led to the notion that the antihyperglycaemic effects of salicylate were related to its uncoupling properties [22, 34]. However, other data led some to wonder as to whether mitochondrial inhibition contributed more to salicylate's side-effects than its therapeutic responses [21,35]. DNP had for example briefly been used earlier in humans as a crude weight loss agent, but its use was stopped when it was found to eliminate "not only the fat but also the patients" [36]. Moreover, in comparative clinical investigation of salicylate and DNP, Reid found that DNP in equivalent dosage had significantly lower effects on blood sugar than aspirin [35], concluding his study by posing the question: "Is it enough to say that the fundamental action of both DNP and salicylate is inhibition of oxidative phosphorylation without further specification to account for quantitative differences?" [35]. In some respects, this debate and the wider concept of mitochondrial inhibition as both a therapeutic and toxic mechanism, is strikingly reminiscent of discussion around metformin action, where inhibition of mitochondrial ATP production by large doses of the drug is thought to account not only for antihyperglycaemic effects, but also sideeffects of the drug such as lactic acidosis [37-40]. Lack of 
hypoglycaemia is another aspect common to both drug types [13, 38]. It is important to note, however, that although both drug classes suppress mitochondrial ATP production, the mitochondrial targets of salicylates and biguanides are different. As described above, salicylate is thought to act on the mitochondria in a similar manner to DNP, which is now known to uncouple mitochondrial ATP production from oxygen consumption by collapsing the proton gradient that couples these two processes. Unlike DNP, biguanides are not true uncouplers because they suppress both ATP production and oxygen consumption by action on the electron transport chain $[37,38,41]$.

\section{Effects on pro-inflammatory IKK-NF-кB signalling}

The first significant enzyme targets of salicylates to be identified were the cyclooxygenase (COX) enzymes, important anti-inflammatory targets of both salicylate and aspirin. In the body, aspirin is rapidly converted to salicylate [42]; however, aspirin does not act simply as a prodrug of salicylate, as aspirin but not salicylate acetylates biomolecules [43]. This property includes irreversible and potent inhibition of COX enzymes through acetylation of an active-site serine [44]. Inhibition of COX-1 in platelets probably accounts for the antithrombotic effect of aspirin to suppress platelet aggregation biomolecules [43]. Salicylate also inhibits COX enzymes less potently by a less well understood mechanism [45]; however, inhibition of COX by either drug occurs at much lower concentrations than are required to elicit antihyperglycaemic effects, suggesting that this aspect of their pharmacology depends on different targets. The first such targets to be proposed followed discovery of salicylate and aspirin's inhibitory effects on inflammatory NF$\kappa \mathrm{B}$ signalling (Figs. 1, 2).

Numerous metabolic stresses that induce insulin resistance and type 2 diabetes stimulate inflammation- and stress-induced kinases such as IKB kinase- $\beta$ (IKK $\beta)$. IKK $\beta$ activates transcription factor nuclear factor $\kappa \mathrm{B}(\mathrm{NF}-\kappa \mathrm{B})$, and obesity is associated with induction of the expression of NF- $\mathrm{\kappa B}$ target genes, such as proinflammatory cytokines, including TNF [46], IL-6 [46, 47] and IL-1 $\beta$ [48] in multiple tissues/organs such as liver and adipose. Salicylate suppresses NF- $\kappa B$ signalling [49, 50], apparently by inhibiting IKK $\beta$, which has been detected in cells and in cell-free experiments [51]. Aspirin and salicylate are both reported to be effective inhibitors of IKK $\beta$ in cell-free experiments [51]. However, these findings need to be interpreted with caution, because more recent kinase profiling experiments published online suggest that salicylate inhibits around $75 \%$ of kinases tested at least as potently as it effects IKK $\beta$. Further investigation linked NF- $\kappa B$ to

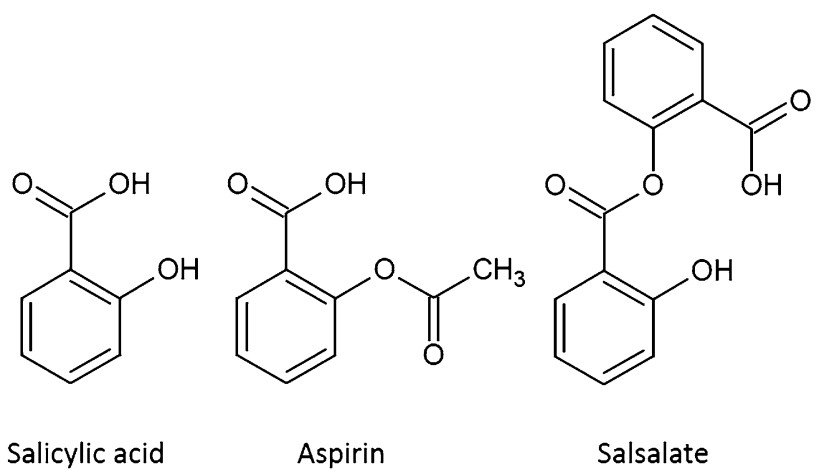

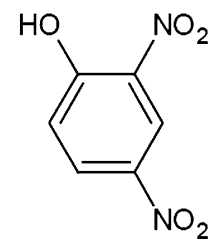

2,4 dinitrophenol<smiles>OCc1ccccc1O[C@@H]1O[C@H](CO)[C@@H](O)[C@H](O)[C@H]1O</smiles>

Salicin
Fig. 1 Structures of salicylic acid and related compounds

antihyperglycaemic effects of salicylate in obese mouse models, on the basis that genetic knockout of the IKK $\beta$ gene produced similar effects to salicylate, protecting against the development of insulin resistance during high fat feeding and in obese $o b / o b$ mice [52].

To understand these effects in more detail, additional experiments found that either salicylate or genetic knockout of the IKK $\beta$ gene prevented lipid-induced insulin resistance, with salicylate normalising skeletal muscle glucose uptake in lipid-infused animals [53]. This supported the idea, reviewed excellently elsewhere [54], that salicylate treats type 2 diabetes at least in part by reducing obesity-dependent chronic inflammation. Tissue-specific knockouts of IKK $\beta$ enabled the contribution of individual organs to be assessed. Blockade of NF- $\mathrm{KB}$ signalling in liver cells was sufficient to preserve insulin sensitivity in the liver, but obesity still induced insulin resistance in other tissues [55]. In contrast, blockade of NF- $\kappa \mathrm{B}$ signalling in myeloid cells protected animals from systemic insulin resistance, underlining the potential importance of inflammatory cell NF- $\mathrm{KB}$ in development of obesity-induced insulin resistance [55]. Data from genetic models is consistent with this concept of salicylate treating diabetes due to its ability to reverse chronic inflammation. In these studies, low-level genetic overexpression of NF- $\kappa \mathrm{B}$ in the liver resulted in type 2 diabetes that could be improved by salicylate, and which was reversed in mice co-expressing the I $\kappa \mathrm{B}$ repressor protein [56]. 


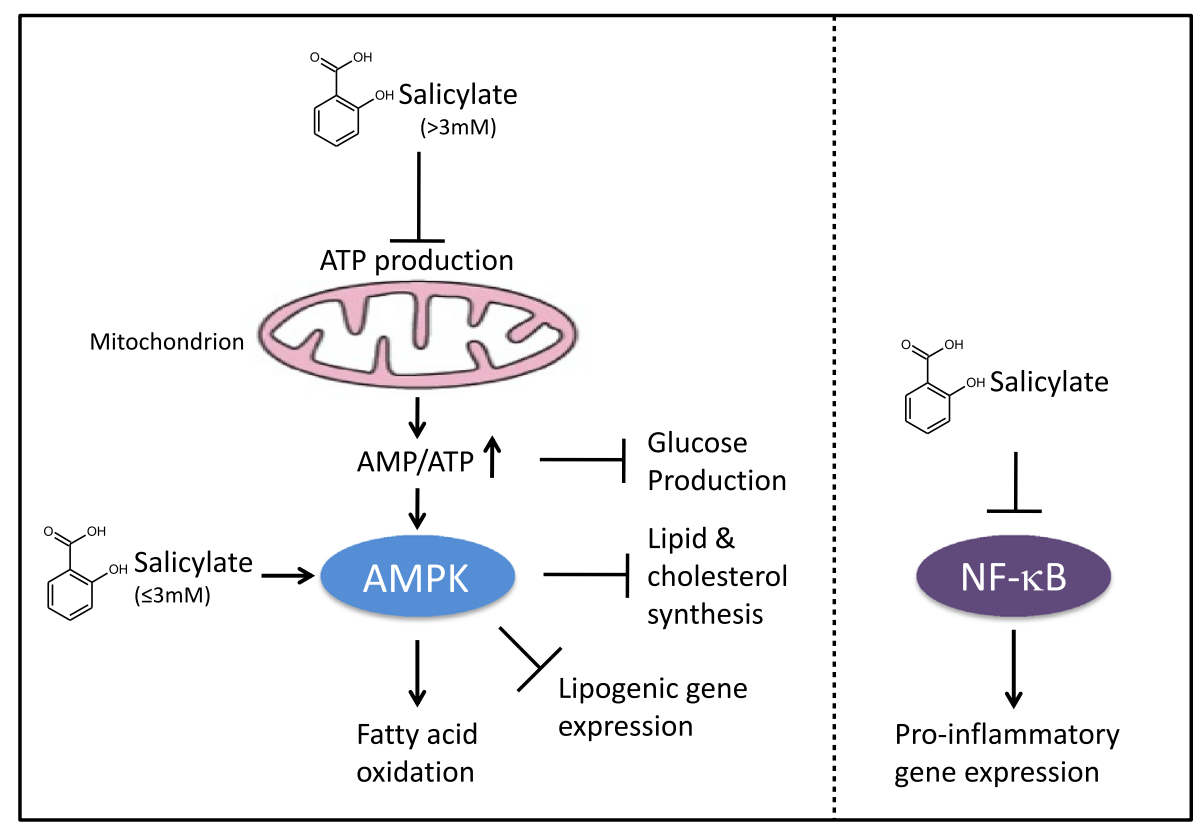

Fig. 2 Schematic illustration of likely antidiabetic targets of salicylate. Salicylate acts on various cells/tissues. In hepatocytes, salicylate (higher concentration $[>3 \mathrm{mM}]$ ) inhibits mitochondrial ATP production, leading to an increase in AMP/ATP ratio, which stimulates AMPK and also would be expected to suppress glucose production via multiple inhibitory effects on gluconeogenic pathways.

\section{Clinical trials}

Given the side effects associated with high-dose salicylate and aspirin, several clinical trials of a salicylate dimer known as salsalate have been carried out on obese nondiabetic subjects $[57,58]$, those with insulin resistance [59], and most recently, with type 2 diabetes [60]. An initial 12-week study using salsalate in patients with type 2 diabetes showed decreased fasting blood glucose, decreased fasting non-esterified fatty acids and increased adipokine expression; however, glucose clearance did not change. Salsalate appears to be well tolerated within the doses used, but side effects such as tinnitus and mild gastrointestinal disturbance were above control groups [59, 60]. In diabetic subjects salsalate caused slight suppression of NF- $\kappa \mathrm{B}$ in subcutaneous abdominal adipose tissue when used at a typical maximum dose of $4 \mathrm{~g} /$ day [60], but in another study there was no effect on NF- $\mathrm{KB}$ in peripheral blood mononuclear cells from patients given $3 \mathrm{~g} /$ day [57]. The modest effects on NF- $\mathrm{KB}$ and glucose clearance might be explained by the fact that the doses of salsalate were lower [57, 60] and/or shorter [57] than the previous work with aspirin (which was used at $7 \mathrm{~g} /$ day for 2 weeks [33]). Salsalate may work at least in part by increasing insulin concentrations, and in this respect salicylate definitely seems to be different from metformin, but such effects may be modest, as hypoglycaemia was only observed in patients
Salicylate at lower concentrations $(\leq 3 \mathrm{mM})$ directly binds and stimulates AMPK. AMPK activation leads to stimulation of fatty acid oxidation and inhibition of lipid synthetic pathways, which would be expected to ameliorate insulin resistance caused by lipotoxicity. Salicylate also inhibits pro-inflammatory responses (e.g., gene expression) through suppression of the NF- $\kappa \mathrm{B}$ pathway

also taking a sulfonylurea [57, 59]. Another interesting difference compared with the earlier work on aspirin is that salsalate did not significantly increase peripheral insulin sensitivity $[33,57,60]$. Consistent with this, salicylate inhibits NF- $\mathrm{\kappa B}$ activity in liver more readily than in muscle, at least in rodents [56]. This suggests that at these intermediate doses, suppression of hepatic glucose production may be more significant than improvements in peripheral insulin sensitivity. The greater significance of hepatic glucose production at lower concentrations of drug is again highly reminiscent of the action of biguanides [37, 38].

\section{Effects on AMPK, a key energy sensor and regulator}

Recent studies have unexpectedly demonstrated that AMPactivated protein kinase (AMPK) is activated by salicylate [61] or aspirin [62] in cultured cells. AMPK is a key cellular energy sensor and regulator of energy homeostasis that coordinates metabolic pathways in order to balance nutrient supply with energy demand. AMPK is activated by various energy stresses that increase intracellular ADP-toATP and AMP-to-ATP ratios, either by promoting ATP consumption (e.g., via muscle contraction) or by reducing ATP generation (e.g., via hypoxia, mitochondrial poisoning) $[63,64]$. Once activated, AMPK functions to restore 
energy balance by accelerating ATP production pathways (e.g., glucose uptake, fatty acid oxidation), while simultaneously suppressing ATP utilization pathways (e.g., lipid, protein synthesis). Because AMPK activation (e.g., by exercise) brings about beneficial physiological outcomes that ameliorate metabolic dysfunctions by improving glycaemic control and plasma lipid profiles, AMPK is considered as a major drug target aimed at treating or delaying the onset of metabolic diseases such as type 2 diabetes [65].

AMPK is a heterotrimeric complex containing a catalytic subunit $(\alpha)$ and two regulatory subunits $(\beta, \gamma)$. Two isoforms of the $\alpha$ - and $\beta$-subunits exist $(\alpha 1, \alpha 2, \beta 1, \beta 2)$, whereas there are three isoforms of the $\gamma$-subunit $(\gamma 1, \gamma 2$, $\gamma 3$ ) [63]. Isoform expression varies among cell types (and also species). AMPK is activated by upstream kinases LKB1 and $\mathrm{Ca}^{2+}$-/calmodulin-dependent protein kinase kinase $\beta(\mathrm{CaMKK} \beta)$ via phosphorylation of Thr172 on the $\alpha$ catalytic subunit. AMP binding to the $\gamma$-subunit allosterically stimulates AMPK, and ADP and/or AMP binding promotes Thr172 phosphorylation and protects it against dephosphorylation by protein phosphatase(s), while ATP competes for binding at the same sites and antagonizes the effects of AMP [64].

In a recent work [61], Hardie and colleagues initially demonstrated that salicylate at concentrations higher than $1 \mathrm{mM}$ stimulated AMPK in HEK293 cells. However, given that a variety of compounds including anti-diabetic drugs (e.g., metformin) and xenobiotics (which are implicated to elicit anti-obesity/diabetic effects such as resveratrol and berberine) activate AMPK in an indirect mechanism by depleting cellular energy levels (i.e., by increasing AMPto-ATP ratio via suppression of mitochondrial respiration) [61], it was perhaps not unexpected that salicylate would be another indirect activator. Interestingly, salicylate activated AMPK in an AMP/ADP-independent manner when treated with concentrations below $10 \mathrm{mM}$ in intact cells [61]. Subsequently, Hardie and colleagues demonstrated [61] that salicylate directly activated recombinant/purified AMPK $\alpha \beta \gamma$ complex in a cell-free assay and was found to bind at the same site as A769662, a small molecule synthetic activator originally identified by Abbott [66]. Indeed, both A769662 and salicylate activate AMPK via allosteric action, and also protect active (phosphorylated) AMPK $\alpha$ at Thr172 from dephosphorylation through interacting with the AMPK $\beta 1$-subunit [67]. To determine if salicylatedependent activation of AMPK had any physiological implications, Hardie and colleagues treated primary hepatocytes with salicylate and observed that it activated AMPK, increased acetyl CoA carboxylase (ACC) phosphorylation (a well characterized AMPK substrate involved in lipid oxidation (ACC2) and synthesis (ACC1)) [68], and fatty acid oxidation in wild-type, but not in AMPK $\beta 1$ - knockout mice [61]. Moreover, administration of salicylate stimulated fat oxidation (as assessed by measuring the respiratory exchange ratio) in vivo in wild-type, and this effect was abolished in the AMPK $\beta 1$-knockout mice [61]. These results verify that the increases in whole-body fat oxidation induced by salicylate were mediated through AMPK activation.

Concentrations of 1-3 mM that were shown to activate AMPK in intact cells [61] are readily reached in the plasma of humans with high doses of either aspirin or salsalate [33, $58,69]$. Recent studies in both rodents and human clinical trials showed that salicylate (mainly its pro-drugs such as salsalate and aspirin) significantly reduced circulating free fatty acids and/or triglycerides in obese/hyperlipidemic rodents [70] and humans with type 2 diabetes [57-60]. These alterations in lipid metabolism are often seen prior to any improvements in insulin sensitivity; therefore, it is tempting to speculate that these lipid-lowering effects by salicylate (or its pro-drugs) might be due the consequences of AMPK activation. However, a long-term salicylate treatment (12 weeks, $250 \mathrm{mg} / \mathrm{kg}$ i.p.) in high-fat diet (HFD)-fed wild-type and AMPK $\beta 1$-knockout mice showed similar protective effects against HFD-induced insulin resistance and glucose intolerance, indicating that effects on AMPK-independent pathways (such as IKK-NFKB) play an important role in the improvement in insulin sensitivity [61]. In addition, the study did not provide detailed metabolic analysis (e.g., lipid metabolism and inflammatory markers), and it would be interesting to compare these parameters between wild-type and AMPK $\beta 1$-knockout mice. Finally, it should also be noted that one study has shown that although $\beta 1$ is the predominant $\beta$ isoform in mouse liver, $\beta 2$ appears to be predominant in human hepatocytes [71]. Given that salicylate stimulates $\beta 1$-containing complex, but not $\beta 2$, this needs to be revisited and clarified in humans (for example, by using primary hepatocytes).

\section{Concluding remarks}

Over 60 years after Reid wondered whether uncoupling properties were really enough to account for the antihyperglycaemic action of salicylate, work in this area has discovered two major additional mechanisms that may contribute towards the improved therapeutic efficacy of this drug over simpler uncouplers. At the high antihyperglycaemic doses of salicylate that promote uncoupling, this drug also regulates NF- $\mathrm{KB}$ and directly activates AMPK in vivo. Emerging clinical data suggest that at intermediate well-tolerated doses, effects on hepatic glucose production may be more significant than those on peripheral insulin sensitivity. Further targeted studies to determine the overall 
contribution of uncoupling, NF- $\mathrm{kB}$, and direct AMPK regulation on each of these antihyperglycaemic physiological responses (as well as anti-inflammatory and antiproliferative/-cancer effects [69]) will allow a deeper understanding of salicylate action to develop.

Conflict of interest The authors declare there is no conflict of interest associated with this manuscript.

\section{References}

1. Duthie GG, Wood AD. Natural salicylates: foods, functions and disease prevention. Food function. 2011;2:515-20.

2. Kramer SN. The Sumerians. Chicago: The University of Chicago Press; 1963

3. Unknown ( $\sim 1500$ B.C.E.) The Edwin Smith surgical papyrus. In: National Library of Medicine translation by J.P. Allen.

4. Leake CD. The Old Egyptian Medical Papyrii. Lawrence: The University of Kansas Press; 1952.

5. Dioscorides P (2000) De Materia Medica: aromatics, translation by T.A. Osbaldeston and R.P.A. Wood. Ibidis Press, Johannesburg.

6. Gross M, Greenberg LA. The Salicylates. New Haven: Hillhouse Press; 1948.

7. Rainsford KD. History and development of the salicylates: Aspirin and related drugs. Boca Raton: Taylor and Francis; 2004. p. $28-51$.

8. MacLagan $\mathrm{T}$. The treatment of acute rheumatism by salicin. Lancet. 1876;107:342-3.

9. Stricker. (1876) Uber die resultate der behandlunger polyarthritis rheumatica mit salicylsaure. Berliner Klinische Wochenshrift 13: $1-2 ; 15-16$; 99-103.

10. Vane JR. Inhibition of prostaglandin synthesis as a mechanism of action for aspirin-like drugs. Nat New Biol. 1971;231:232-5.

11. Ebstein W. Zur Therapie des Diabetes mellitus, insbesondere uber die Anwendung des salicylsauren Natron bei demselben. Ber Klin Woch. 1876;13:337-40.

12. Williamson RT. On the treatment of glycosuria and diabetes mellitus with sodium salicylate. Brit Med J. 1901;1:760-2.

13. Reid J, MacDougall AI, Andrews MM. Aspirin and diabetes mellitus. Brit Med J. 1957;2:1071-4.

14. Ingle DJ. Effect of aspirin upon glycosuria of the partially depancreatized rat. Proc Soc Exp Biol Med NY. 1950;75:673.

15. Smith MJH, Meade BW. The effect of salicylate on glycosuria, blood glucose and liver glycogen of the alloxan-diabetic rat. Biochem J. 1952;51:18-20.

16. Reid J, Lightbody TD. The insulin equivalence of salicylate. Brit Med J. 1959;1:897-900.

17. Cochran JB. The respiratory effects of salicylate. Brit Med J. 1952;2:964-7.

18. Meade BW. Effect of certain hydroxybenzoic acids on the oxygen consumption of Wistar rats. Ann Rheum Dis. 1954;13:60-2.

19. Smith MJH, Jeffrey SW. The effects of salicylate in oxygen consumption and carbohydrate metabolism in the isolated rat diaphragm. Biochem J. 1956;63:524-8.

20. Smith MJH, Jeffrey SW. The effects of salicylate on creatine phosphate and adenosine triphosphate in the isolated diaphragm. Biochem J. 1956;64:589-92.

21. Brody T. Action of sodium salicylate and related compounds on tissue metabolism in vitro. J Pharmacol Exp Ther. 1956;117: 39-51.

22. Jeffrey SW, Smith MJH. Some effects of salicylate on mitochondria from rat liver. Biochem J. 1959;72:462-5.
23. Smith MJH, Moses V. Uncoupling reagents and metabolism. 1. Effects of salicylate and 2,4-dinitrophenol on the incorporation of $14 \mathrm{C}$ from labelled glucose and acetate into the soluble intermediates of isolated rat tissues. Biochem J. 1960;76:579-85.

24. Barnes JM, Duff JI, Threlfall CJ. The behaviour of mammalian striated muscle in the presence of 2:4-dinitrophenol. J Physiol. 1955;130:585-600.

25. Ottaway JH. The effect of insulin on the anaerobic metabolism of rat diaphragm. Biochem J. 1955;61:441-7.

26. Sacks J, Sinex FM. The effect of 2,4-dinitrophenol on the turnover of the acid-soluble phosphorus of rat diaphragm. Arch Biochem Biophys. 1952;39:205-13.

27. Fang V, Foye WO, Robinson SM, Jenkins HJ. Hypoglycemic activity and chemical structure of the salicylates. J Pharmaceutical Sci. 1968;57:2111-6.

28. Reid J, Watson RD, Cochran JB, Sproull DH. Sodium gammaresorcylate in rheumatic fever. Brit Med J. 1951;2:321-6.

29. Manchester KL, Randle PJ, Smith GH. Some effects of sodium salicylate on muscle metabolism. Brit Med J. 1958;1:1028-30.

30. Randle PJ, Smith GH. Regulation of glucose uptake by muscle. 2. The effects of insulin, anaerobiosis and cell poisons on the penetration $\mathrm{f}$ isolated rat diaphragm by sugars. Biochem J. 1958;70: 501-8.

31. Huggins AK, Smith MJH. Uncoupling reagents and metabolism. 5. Effects of salicylate and 2,4,-dinitrophenol on the metabolism of isolated rat diaphragm. BiochemJ. 1962;85:394-402.

32. Woods HF, Stubbs WA, Johnson G, Alberti KGMM. Inhibition by salicylate of gluconeogenesis in the isolated perfused rat liver. Clin Exp Pharmacol Physiol. 1974;1:535-40.

33. Hundal RS, Peterson KF, Mayerson AB, et al. Mechanism by which high-dose aspirin improves glucose metabolism in type 2 diabetes. J Clin Invest. 2002;109:1321-6.

34. Mahler RF. Chemistry of Hypoglycaemic Substances Other than Insulin. Brit Med Bull. 1960;16:250-4.

35. Reid J. Dinitrophenol and diabetes mellitus: a comparison with salicylate. Brit Med J. 1958;2:724-7.

36. Racker E. A new look at mechanisms in bioenergetics. New York: Academic Press; 1976.

37. Rena G, Pearson ER, Sakamoto K. Molecular mechanism of action of metformin: old or new insights? Diabetologia. 2013;56:1898-906.

38. Rena G, Pearson ER, Sakamoto K. Molecular action and pharmacogenetics of metformin: current understanding of an old drug. Diabetes Manag. 2012;2:439-52.

39. Owen MR, Doran E, Halestrap AP. Evidence that metformin exerts its anti-diabetic effects through inhibition of complex 1 of the mitochondrial respiratory chain. Biochem J. 2000;348: $607-14$.

40. Foretz M, Hébrard S, Leclerc J, et al. Metformin inhibits hepatic gluconeogenesis in mice independently of the LKB1/AMPK pathway via a decrease in hepatic energy state. J Clin Invest. 2010;120:2355-69.

41. Falcone AB, Mao RL, Shrago E. A study of the action of hypoglycemia-producing biguanide and sulfonylurea compounds on oxidative phosphorylation. J Biol Chem. 1962;237:904-9.

42. Graham GG, Roberts MS, Day RO, Rainsford KD. Pharmacokinetics and metabolism of the salicylates aspirin and related drugs. Boca Raton: Taylor and Francis; 2004. p. 121-80.

43. Rainsford KD. Pharmacology and biochemistry of salicylates and related drugs. Aspirin and related drugs. Boca Raton: Taylor and Francis; 2004. p. 236-375.

44. DeWitt DL, el-Harith EA, Kraemer SA, et al. The aspirin and heme-binding sites of ovine and murine prostaglandin endoperoxide synthases. J Biol Chem. 1990;265:5192-8.

45. Mitchell JA, Saunders M, Barnes PJ, Newton R, Belvisi MG. Sodium salicylate inhibits cyclo-oxygenase-2 activity 
independently of transcription factor (nuclear factor $\kappa \mathrm{B}$ ) activation: role of arachidonic acid. Mol Pharmacol. 1997;51:907-12.

46. Hotamisligil GS, Arner P, Caro JF, Atkinson RL, Spiegelman $\mathrm{BM}$. Increased adipose tissue expression of tumor necrosis factoralpha in human obesity and insulin resistance. J Clin Invest. 1995;95:2409-15.

47. Park EJ, Lee JH, Yu G-Y, et al. Dietary and genetic obesity promote liver inflammation and tumorigenesis by enhancing IL-6 and TNF expression. Cell. 2010;140:197-208.

48. Moschen AR, Molnar C, Enrich B, Geiger S, Ebenbichler CF. Adipose and liver expression of interleukin (IL)-1 family members in morbid obesity and effects of weight loss. Mol Med. 2011;17:840-5.

49. Pierce JW, Read MA, Ding H, Luscinskas FW, Collins T. Salicylates inhibit I kappa B-alpha phosphorylation, endothelialleukocyte adhesion molecule expression, and neutrophil transmigration. J Immunol. 1996;156:3961-9.

50. Kopp E, Ghosh S. Inhibition of NF-kB by sodium salicylate and aspirin. Science. 1994;265:956-9.

51. Yin M-J, Yamamoto Y, Gaynor RB. The anti-inflammatory agents aspirin and salicylate inhibit the activity of I kappa B kinase-beta. Nature. 1998;396:77-80.

52. Yuan M, Konstantopoulos N, Lee J, et al. Reversal of obesity and diet-induced insulin resistance with salicylates or targeted disruption of Ikk beta. Science. 2001;293:1673-7.

53. Kim JK, Kim Y-J, Fillmore JJ, et al. Prevention of fat-induced insulin resistance by salicylate. J Clin Investig. 2001;108:437-46.

54. Shoelson SE, Lee J, Goldfine AB. Inflammation and insulin resistance. J Clin Invest. 2006;116:1793-801.

55. Arkan MC, Hevener AL, Greten FR, et al. IKK-[beta] links inflammation to obesity-induced insulin resistance. Nat Med. 2005;11:191-8.

56. Cai D, Yuan M, Frantz DF, et al. Local and systemic insulin resistance resulting from hepatic activation of IKK-[beta] and NF-[kappa]B. Nat Med. 2005;11:183-90.

57. Koska J, Ortega E, Bunt JC, et al. The effect of salsalate on insulin action and glucose tolerance in obese non-diabetic patients: results of a randomised double-blind placebo-controlled study. Diabetologia. 2009;52:385-93.

58. Fleischman A, Shoelson SE, Bernier R, Goldfine AB. Salsalate improves glycemia and inflammatory parameters in obese young adults. Diabetes Care. 2008;31:289-94.
59. Goldfine AB, Fonseca V, Jablonski KA, Pyle L, Staten MA, Shoelson SE. The effects of salsalate on glycemic control in patients with type 2 diabetes. Ann Intern Med. 2010;152:346-57.

60. Goldfine AB, Conlin PR, Halperin F, et al. A randomised trial of salsalate for insulin resistance and cardiovascular risk factors in persons with abnormal glucose tolerance. Diabetologia. 2013;56:714-23.

61. Hawley SA, Fullerton MD, Ross FA, et al. The ancient drug salicylate directly activates AMP-activated protein kinase. Science. 2012;336:918-22.

62. Din FV, Valanciute A, Houde VP, et al. Aspirin inhibits mTOR signalling, activates AMP-activated protein kinase, and induces autophagy in colorectal cancer cells. Gastroenterology. 2012; 142(1504-1515):e1503.

63. Steinberg GR, Kemp BE. AMPK in health and disease. Physiol Rev. 2009;89:1025-78.

64. Hardie DG, Ross FA, Hawley SA. AMPK: a nutrient and energy sensor that maintains energy homeostasis. Nat Rev Mol Cell Biol. 2012;13:251-62.

65. Hardie DG, Ross FA, Hawley SA. AMP-activated protein kinase: a target for drugs both ancient and modern. Chem Biol. 2012;19:1222-36.

66. Cool B, Zinker B, Chiou W, et al. Identification and characterization of a small molecule AMPK activator that treats key components of type 2 diabetes and the metabolic syndrome. Cell Metab. 2006;3:403-16.

67. Xiao B, Sanders MJ, Carmena D, et al. Structural basis of AMPK regulation by small molecule activators. Nat commun. 2013;4: 3017.

68. Fullerton MD, Galic S, Marcinko K, et al. Single phosphorylation sites in Acc1 and Acc2 regulate lipid homeostasis and the insulinsensitizing effects of metformin. Nat Med. 2013;19:1649-54.

69. Steinberg GR, Dandapani M, Hardie DG. AMPK: mediating the metabolic effects of salicylate-based drugs? Trends Endocrinol Metab TEM. 2013;24:481-7.

70. van Diepen JA, Vroegrijk IO, Berbee JF, et al. Aspirin reduces hypertriglyceridemia by lowering VLDL-triglyceride production in mice fed a high-fat diet. Am J Physiol Endocrinol Metab. 2011;301:E1099-107.

71. Stephenne X, Foretz M, Taleux N, et al. Metformin activates AMP-activated protein kinase in primary human hepatocytes by decreasing cellular energy status. Diabetologia. 2011;54:3101-10. 